CARDIOVASCULAR MEDICINE

\title{
Angiogenesis, thrombogenesis, endothelial dysfunction and angiographic severity of coronary artery disease
}

\author{
N A Chung, C Lydakis, F Belgore, F L Li-Saw-Hee, A D Blann, G Y H Lip
}

Heart 2003;89:1411-1415

See end of article for authors' affiliations

Correspondence to:

Professor G Y H Lip,

Haemostasis Thrombosis and Vascular Biology Unit, University Department of

Medicine, City Hospital, Birmingham B18 7QH, UK; g.y.h.lip@bham.ac.uk

Accepted 5 June 2003
Background: Thrombogenesis, angiogenesis, and endothelial damage/dysfunction are components in the pathogenesis of atherosclerosis.

Objective: To investigate the relation of these variables to atherosclerotic disease severity and the possible interrelations between the three.

Methods: 111 patients attending for coronary angiography were studied (85 male, 26 female; mean (SD) age, 61.6 (10.0) years). Plasma concentrations of von Willebrand factor (vWf, a marker of endothelial damage/dysfunction), vascular endothelial growth factor (VEGF, associated with angiogenesis), soluble VEGF receptor Flt- 1 (sFlt-1), and tissue factor (TF, a key component of coagulation) were measured by an enzyme linked immunosorbent assay. Following angiography, disease severity was assessed by the number of coronary vessels diseased ( $>50 \%$ stenosis) and by a coronary atheroma score.

Results: All indices were raised in the patients compared with 34 healthy controls except sFlt- 1 , which was lower in the patients. No significant correlations were found between the coronary atheroma score and values of $\mathrm{vWf}$ (Spearman correlations: $r=0.21, p=0.83)$, VEGF $(r=0.11, p=0.27$ ), or TF $(r=-0.04, p=0.68)$. However, there was an inverse correlation between plasma sFlt- 1 and coronary atheroma score $(r=-0.19, \mathrm{p}=0.049)$. The number of vessels diseased had no relation to any marker. Correlations were found between TF and VEGF $(r=0.25, p=0.008)$ and between TF and sFlt- 1 $(r=0.42, \mathrm{p}<0.001)$ in the patients.

Conclusions: Despite evidence of abnormal angiogenesis (VEGF and sFlt-1), thrombogenesis (TF), and endothelial damage/dysfunction ( $\mathrm{vWf}$ ) in the patients with coronary artery disease, there was no correlation between VEGF, sFlt-1, vWf, or TF and angiographically defined disease severity.
A therosclerosis is the pathophysiological process underlying coronary artery disease. Roles for thrombogenesis and angiogenesis have been widely reported to be involved in the development of atherosclerosis. A close link between angiogenesis and thrombogenesis has been clearly shown in cancer biology, ${ }^{1-3}$ but there are limited data on this link in atherosclerosis. Moreover, thrombosis and angiogenesis may be related to disturbances of endothelial cell physiology and thus a link between the three processes of angiogenesis, thrombogenesis, and endothelial disturbance is plausible.

In atherosclerosis, angiogenesis within the adventitia of arterial walls is seen in the development of plaques, and extends into the media and intima as the lesions progress. ${ }^{45}$ Furthermore, the expression of vascular endothelial growth factor (VEGF), an essential component in angiogenesis, has been positively correlated with the number of intimal blood vessels found within atherosclerotic plaques. ${ }^{6}$

Plasma concentrations of VEGF and a soluble form of its receptor Flt-1 (sFlt-1) are quantifiable by an enzyme linked immunosorbent assay (ELISA). ${ }^{7}$ Plasma concentrations of both VEGF and sFlt- 1 are abnormal in patients with coronary artery disease and peripheral vascular disease. ${ }^{8-10}$ Raised concentrations of VEGF have also been found in patients with risk factors for coronary artery disease, such as hypertension and hyperlipidaemia, but with no clinically overt disease. ${ }^{11} 12$

Tissue factor (TF) is one of the key components in the coagulation cascade. In atherosclerosis, raised plasma concentrations of TF are found in patients with ischaemic heart disease (in unstable angina, stable angina, and patients with a history of previous myocardial infarction) when compared with healthy controls. ${ }^{13}$ These increases in VEGF and TF could be mediated through a dysfunctional endothelium that is associated with the atherosclerotic process. Indeed, both VEGF and TF are expressed by endothelial cells. The plasma marker von Willebrand factor (vWf, also essentially a procoagulant in promoting thrombosis) is a good index of endothelial cell damage/dysfunction, and raised vWf concentrations have been reported in numerous cardiovascular conditions (as well as in many cancers) and predict poor outcome-including myocardial infarction, stroke, and death-in prospective studies. ${ }^{14-16}$

We hypothesised that angiogenesis, thrombogenesis, and endothelial damage are related to disease severity in patients with coronary artery disease, and furthermore could be related to each other as well as to disease severity in this condition. To test these hypotheses, we measured plasma VEGF, sFlt-1, TF, and vWf in patients with coronary artery disease.

\section{METHODS}

We studied 111 patients (85 male, 26 female, mean (SD) age 61.6 (10) years) attending for elective day case coronary angiography. All patients had a history of stable angina. We excluded patients with recent myocardial infarction (within the last six weeks), unstable angina, stroke, or congestive heart failure, as well as those with renal or liver impairment or on warfarin treatment. Following the procedure all angiograms were analysed for disease severity (see below). The West Birmingham ethics

Abbreviations: $s F l t-1$, soluble VEGF receptor Flt- 1 ; TF, tissue factor; VEGF, vascular endothelial growth factor; VWf, von Willebrand factor 
committee passed the protocol, and informed consent was obtained.

Baseline results were compared with 34 age and sex matched healthy controls ( 24 male, 10 female, mean age 59.4 (12) years), recruited from hospital staff and preoperative clinics for minor procedures, including hernia repairs, cataract surgery, and so on. All healthy controls were "healthy" on the basis of careful clinical history and examination, as well as basic blood screening tests. These subjects were included to provide a perspective (that is, what should be "healthy" values) for the patient data; no direct case-control comparison is intended.

\section{Blood samples and analysis}

Citrated plasma samples were taken before angiography and immediately placed in ice before being centrifuged at $1000 \mathrm{~g}$ for 20 minutes at $4{ }^{\circ} \mathrm{C}$. They were then stored at $-70^{\circ} \mathrm{C}$ until the time of analysis. Samples were analysed by an in-house ELISA for VEGF and sFlt-1 (R\&D Systems, Abingdon, UK) (previously described in $\operatorname{detail}^{7}$ ), vWf (Dako, Ely, UK), and TF (Axis-Shield, Dundee, UK). The lower limits of detection by ELISA were $10 \mathrm{pg} / \mathrm{ml}$ for VEGF and TF, $0.1 \mathrm{ng} / \mathrm{ml}$ for sFlt, and $2 \mathrm{IU} / \mathrm{dl}$ for vWf. The interassay and intra-assay variabilities were $<5 \%$ and $10 \%$, respectively, for all assays.

\section{Coronary angiography and analysis}

Coronary angiography was undertaken by the percutaneous transfemoral approach and images were recorded digitally. All angiograms were analysed by a single experienced cardiologist who was blinded to the clinical details of the patients. Disease severity was assessed in two ways: first, by the number of vessels $(0-3)$ with at least one significant stenosis ( $>50 \%$ stenosis); and second, by an atheroma score (the coronary atheroma score, as previously described ${ }^{17}$ ) to indicate disease severity. In brief, 15 proximal segments of the major coronary arteries were examined, as described by an ad hoc committee on grading of coronary artery disease of the Council on Cardiovascular Surgery, American Heart Association. ${ }^{18}$ Atheromatous lesions in each segment were evaluated for extension (number of plaques) and size (\% of vessel diameter involved). The scores for extension and size were multiplied together for each segment and the total score was calculated as the sum of individual segment scores divided by the number of segments analysed. Segments distal to a total occlusion were not analysed. Thus patients could be classed as having "0 vessels diseased" with no stenoses of $>50 \%$, but still have an atheroma score above 0 , as the coronary atheroma score includes lesions causing less than $50 \%$ stenosis in the analysis.

\section{Power calculations}

Our primary hypothesis was a significant correlation between the coronary atheroma score (a continuously variable index) and any one of the plasma markers. Given the variability of the measured indices and the coronary atheroma score, we believe that a Spearman correlation coefficient of 0.35 is meaningful. To achieve this, for a two sided probability of $<0.01$ and $1-\beta$ of $0.85,100$ datasets are required. ${ }^{19}$ Thus to be fully confident in our data we recruited in excess of this figure. The number of diseased arteries is distributed as a discrete but ordered dataset. We hypothesised increasing differences in vWf, VEGF, and TF, but decreasing sFlt-1, as disease became more severe. Such differences were sought using the Kruskal-Wallis test. The number of subjects with coronary artery disease compared with controls provided the power to detect a difference of 0.5 of a standard deviation at $\mathrm{p}<0.05$ and $1-\beta=0.80$.

\section{Statistical methods}

Statistical analyses were done using the statistical program SPSS 10.0 for Windows. Parametric results are expressed as mean (SD) and differences between groups were compared by unpaired Student's $t$ test. Non-categorical data were compared by the $\chi^{2}$ test. Non-parametric results are expressed as medians with interquartile ranges (IQR) and comparisons made using Mann-Whitney and Kruskal-Wallis tests. Multiple regression analyses were used to determine predictors for the research indices. Correlations were examined using Spearman's rank correlation. The level of significance was taken to be $\mathrm{p}<0.05$.

\section{RESULTS}

\section{Cross sectional data}

Demographic details, risk factors, and drugs prescribed for the patients with coronary artery disease are summarised in table 1. vWf, VEGF, sFlt-1, and TF data are presented in table 2. As expected, all indices were increased in the patients with coronary artery disease compared with the healthy controls except for sFlt-1, which was lower in the patients.

\section{Relation to disease severity}

The distribution of coronary atheroma score was not normally distributed (median 1.77 (IQR $0.23-4.5$ ). No significant Spearman correlations were found between coronary atheroma score and concentrations of vWf $(r=0.21, \mathrm{p}=0.83)$, VEGF $(r=0.11, \mathrm{p}=0.27)$, and TF $(r=-0.04, \mathrm{p}=0.68)$. However, there was an inverse correlation between sFlt-1 and coronary atheroma score $(r=-0.19, \mathrm{p}=0.049)$.

When coronary angiograms were analysed by the number of vessels with significant stenoses, the cohort was divided as follows:

- 37 patients (33\%) had no vessels significantly diseased (with $>50 \%$ stenosis)

- 27 patients (24\%) had one vessel diseased

- 22 patients (20\%) had two vessels diseased

- 25 patients (23\%) had three vessels diseased.

There were no significant differences in plasma concentrations of vWf, VEGF, sFlt-1, and TF between these four groups (table 3$)$. Note that patients with no vessels with significant stenoses had a median coronary atheroma score of 1.42 (IQR $0.67-2.40$ ) and concentrations of vWf, VEGF, and TF were higher in these patients than in the control group $(p<0.001$, $\mathrm{p}=0.014$, and $\mathrm{p}=0.020$, respectively).

\section{Subgroups and multiple regression analyses}

The patients with coronary artery disease were then subdivided according to the presence or absence of the risk factors diabetes mellitus, hypertension, and hyperlipidaemia. The presence or absence of diabetes or hyperlipidaemia made no significant difference to plasma concentrations of VEGF, sFlt-1, TF, or vWf (data not shown). Only patients with coronary artery disease and hypertension had significantly higher concentrations of TF $(p=0.009)$ when compared with normotensive patients (data not shown).

When adjusting for clinical indices in the coronary artery disease patients using standard multiple regression analyses with plasma concentrations of VEGF, sFlt-1, TF, or vWf as dependent variables, age was found to be a predictor for VEGF concentrations $(\beta=-0.190, p=0.045)$, and HDL values a predictor for sFlt-1 concentrations $(\beta=0.300$, $p=0.003)$. However, the relatively small numbers involved in these analyses must be borne in mind when interpreting their relevance. 
Table 1 Demographic characteristics of patients with coronary artery disease and healthy controls

\begin{tabular}{|c|c|c|c|}
\hline & Patients & Controls & p Value \\
\hline Age (years) (mean (SD)) & $61.6(10)$ & 59.4 (12) & 0.30 \\
\hline Male (\%) & 77 & 71 & 0.48 \\
\hline Smokers (\%) & 28 & 15 & 0.15 \\
\hline \multicolumn{4}{|l|}{ Past medical history (n (\%)) } \\
\hline Previous myocardial infarction & $36(32.4)$ & & \\
\hline Hypertension & $40(36)$ & & \\
\hline Diabetes & 15 (13.5) & & \\
\hline \multicolumn{4}{|l|}{ Drugs (n (\%)) } \\
\hline Aspirin & $82(73.9)$ & & \\
\hline Thiazide & $7(6.3)$ & & \\
\hline Diuretic & $24(21.6)$ & & \\
\hline ACE inhibitor & $27(24.3)$ & & \\
\hline$\beta$ Blocker & $61(55)$ & & \\
\hline Calcium channel blocker & $54(48.6)$ & & \\
\hline Nitrate & 57 (51.4) & & \\
\hline Glyceryl trinitrate & $43(38.7)$ & & \\
\hline Systolic blood pressure (mm Hg) (mean (SD)) & $139(23)$ & 135 (17) & 0.30 \\
\hline Diastolic blood pressure (mm Hg) (mean (SD)) & 79 (11) & $85(10)$ & 0.02 \\
\hline Total cholesterol (mmol/l) (mean (SD)) & $5.5(0.9)$ & $5.7(1.0)$ & 0.20 \\
\hline Triglycerides (mmol/l) (mean (SD)) & $1.9(1.4)$ & $1.3(0.3)$ & 0.009 \\
\hline $\mathrm{HDL}(\mathrm{mmol} / \mathrm{l})($ mean $(\mathrm{SD}))$ & $1.2(0.3)$ & $1.3(0.4)$ & 0.14 \\
\hline LDL (mmol/l) (mean (SD)) & $3.4(0.8)$ & $3.8(1.1)$ & 0.07 \\
\hline
\end{tabular}

\section{Relations between indices}

Concentrations of VEGF correlated with TF in the patients with coronary artery disease $(r=0.25, \mathrm{p}=0.008)$, while concentrations of sFlt-1 correlated with VEGF $(r=0.36$, $\mathrm{p}<0.001)$ and TF $(r=0.42, \mathrm{p}<0.001)$. No other correlations reached significance.

\section{DISCUSSION}

This study confirms previous observations of higher plasma VEGF, TF, and vWf in patients with coronary artery disease compared with healthy controls. ${ }^{8}{ }^{13} 20-23$ We found no relation between the coronary atheroma score and concentrations of VEGF, sFlt-1, TF, or vWf; furthermore, there were no significant differences in concentrations of indices according to subgroups, based on the number of vessels with $>50 \%$ stenosis in the patient group. Furthermore, the (very) weak Spearman correlation coefficient of -0.19 between coronary atheroma score and sFlt $(\mathrm{p}=0.049)$ has to be interpreted with caution. The possibility arises that the finding of higher concentrations of vWf, VEGF, and TF in patients undergoing angiography with no coronary artery stenoses of more than $50 \%$ compared with healthy controls may be explained by the presence of risk factors (hypertension, diabetes, and hypercholesterolaemia) in this patient group.

We are unaware of other studies investigating the relation between coronary atheroma score or peripheral plasma VEGF concentrations and coronary artery disease severity. The lack of correlation between plasma VEGF and coronary artery disease severity may reflect the more generalised nature of thrombogenesis, angiogenesis, and endothelial disturbance, rather than that occurring in specific vascular beds. In one study looking at coronary collateral flow and intracoronary VEGF concentrations, Fleisch and colleagues found a trend toward higher concentrations of intracoronary VEGF with more extensive coronary artery disease, as assessed by the number of diseased coronary arteries (stenosis $>50 \%$ ) on coronary angiography. ${ }^{24}$ However, concentrations of VEGF were measured on serum samples, and there is increasing evidence that VEGF should be measured on plasma samples, as in our study. 25

In the present study, we did not find any significant relation between vWf and coronary artery disease severity. We accept that there are many possible indices of endothelial damage/dysfunction, and in our study we chose to measure vWf, the most well established index of endothelial damage/ dysfunction. ${ }^{20}$ Indeed, in another study, ${ }^{27}$ Yildirir and colleagues measured concentrations of E-selectin, VCAM-1, and ICAM-1 as markers of endothelial cell activation ${ }^{20}$ in 83 consecutive patients attending for coronary angiography. They used patients with no coronary artery stenoses of $>50 \%$ as their control group and found significantly lower concentrations of VCAM-1 and E-selectin in their controls compared with patients with one or more stenoses of $>50 \%$. However, included among their subjects were patients with unstable angina $(\mathrm{n}=23)$, whereas in the present study we excluded all patients with episodes of unstable angina within the previous six weeks. A significant correlation between severity of coronary lesions, as graded using the Gensini score, and E-selectin concentrations was only found in this small subgroup of patients with unstable angina, and there

$\begin{aligned} & \text { Table } 2 \text { Plasma concentrations of von Willebrand factor, vascular endothelial growth } \\
& \text { factor, soluble Flt-1, and tissue factor in patients with coronary artery disease and controls }\end{aligned}$
\begin{tabular}{llll}
\multicolumn{5}{l}{$\begin{array}{l}\text { Coronary artery disease } \\
\text { patients }\end{array}$} & Healthy controls & p Value \\
\hline vWf (IU/dl) & $128(103-143)$ & $90(82-101)$ & $<0.001$ \\
VEGF (pg/ml) & $130(100-250)$ & $80(20-176)$ & 0.002 \\
sFlt (ng/ml) & $7.5(1.9-19)$ & $20(9-40)$ & 0.003 \\
TF (pg/ml) & $90(10-230)$ & $60(10-100)$ & 0.01
\end{tabular}

Values are median (interquartile range), analysed by Mann-Whitney test.

sFlt-1, soluble Flt-1; TF, tissue factor; VEGF, vascular endothelial growth factor; WWf, von Willebrand factor. 
Table 3 Concentrations of von Willebrand factor, vascular endothelial growth factor, soluble Flt-1, and tissue factor in relation to number of coronary arteries diseased

\begin{tabular}{llllll}
\hline \multicolumn{7}{l}{ Number of diseased coronary arteries } \\
\cline { 2 - 6 } & $\mathbf{0}$ & $\mathbf{1}$ & $\mathbf{2}$ & $\mathbf{3}$ & p Value \\
\hline Number of patients & 37 & 27 & 22 & 25 & \\
vWf (IU/dl) & $133(105-143)$ & $123(103-141)$ & $118(93-146)$ & $132(106-148)$ & 0.076 \\
VEGF (pg/ml) & $130(100-540)$ & $120(100-200)$ & $135(94-240)$ & $160(100-260)$ & 0.958 \\
sFlt $(\mathrm{ng} / \mathrm{ml})$ & $9.5(3-21)$ & $12.2(2.5-23)$ & $6.5(1.6-16)$ & $5.0(1-15)$ & 0.182 \\
TF $(\mathrm{pg} / \mathrm{ml})$ & $90(10-585)$ & $100(10-230)$ & $90(14.5-375)$ & $80(12.5-190)$ & 0.118 \\
\hline
\end{tabular}

Values are median (interquartile range), analysed by the Kruskal-Wallis test.

sFlt-1, soluble Flt-1; TF, tissue factor; VEGF, vascular endothelial growth factor; VWf, von Willebrand factor.

were no significant correlations between ICAM-1 or VCAM-1 and disease severity.

We are unaware of any studies to date that have investigated concentrations of TF in relation to angiographic disease severity, although no significant relation was present. Nonetheless, we have shown a correlation between TF and concentrations of the angiogenic markers VEGF and sFlt-1, suggesting a link between angiogenesis and thrombogenesis. However, the relevance of this observation should be interpreted with caution given the low values of the Spearman correlation coefficients $(r=0.225-0.42)$. Further studies with larger sample sizes are needed to confirm the hypothesis that angiogenesis and thrombogenesis are closely related in vascular disease, as appears to be the case in embryonic studies ${ }^{28}$ and cancer. ${ }^{29-33}$

\section{Study limitations}

Our study was limited by its cross sectional design, but we have attempted to relate our research indices to coronary artery disease severity using two different methods of scoring disease severity. In addition, we recognise that our markers may simply reflect generalised vascular disease or risk factors such as diabetes or hypertension (rather than coronary artery disease per se, or concomitant treatment), and this would be a limitation common to all studies examining plasma markers such as VEGF, TF, and vWf in patients with coronary artery disease compared with healthy controls. ${ }^{8}{ }^{13} 20-23$ Furthermore, acute episodes of ischaemia could influence the production of angiogenic factors, ${ }^{34-37}$ but we only included patients with chronic stable symptoms.

\section{Conclusions}

Despite evidence of abnormal angiogenesis (VEGF and sFlt$1)$, thrombogenesis (TF), and endothelial damage/dysfunction (vWf) in our patients with coronary artery disease, there was no meaningful relation between VEGF, sFlt-1, vWf, or TF and angiographically defined coronary artery disease severity.

\section{ACKNOWLEDGEMENTS}

We acknowledge the support of the City Hospital Research and Development Programme for the Haemostasis Thrombosis and Vascular Biology Unit. NAC is supported by a non-promotional research fellowship from Merck Sharpe and Dohme.

\section{Authors' affiliations}

N A Chung, C Lydakis, F Belgore, F L Li-Saw-Hee, A D Blann, G Y H Lip, Haemostasis Thrombosis and Vascular Biology Unit, University Department of Medicine, City Hospital, Birmingham, UK

\section{REFERENCES}

1 Donati MB, Falanga A. Pathogenetic mechanisms of thrombosis in malignancy. Acta Haematol 2001;106:18-24.

2 Francis JL. Haemostasis and cancer. Med Lab Sci 1989;46:331-46.

3 Mackman N. Gene targeting in hemostasis. tissue factor. Front Biosci 2001:6:D208-15.
4 Kamat BR, Galli SJ, Barger AC, et al. Neovascularization and coronary atherosclerotic plaque: cinematographic localization and quantitative histologic analysis. Hum Pathol 1987;18:1036-42.

5 Moulton KS. Plaque angiogenesis and atherosclerosis. Curr Atheroscler Rep 2001;3:225-33.

6 Chen YX, Nakashima Y, Tanaka K, et al. Immunohistochemical expression of vascular endothelial growth factor/vascular permeability factor in atherosclerotic intimas of human coronary arteries. Arterioscler Thromb Vasc Biol 1999;19:131-9.

7 Belgore FM, Blann AD, Lip GYH. Measurement of free and complexed soluble vascular endothelial growth factor receptor, Flt-1, in fluid samples: development and application of two new immunoassays. Clin Sci 2001; 100:567-75.

8 Belgore $\mathrm{F}$, Lip $\mathrm{GYH}, \mathrm{McCollum} \mathrm{CN}$, et al. Vascular endothelial growth factor (VEGF) and soluble VEGF receptor (sFlt-1) levels in coronary artery disease and peripheral artery disease. Blood Coagul Fibrinolysis 1999;10:536.

9 Ogawa H, Suefuji H, Soejima H, et al. Increased blood vascular endothelial growth factor levels in patients with acute myocardial infarction. Cardiology 2000:93:93-9.

10 Roller RE, Renner W, Dorr A, et al. Oxidative stress and increase of vascular endothelial growth factor in plasma of patients with peripheral arterial occlusive disease. Thromb Haemost 2001;85:368.

11 Belgore FM, Lip GYH, Blann AD. Successful therapy reduces levels of vascular endothelial growth factor (VEGF) in patients with hypertension and patients with hypercholesterolemia. Atherosclerosis 2000;151:599.

12 Belgore FM, Blann AD, Li-Saw-Hee FL, et al. Plasma levels of vascular endothelial growth factor and its soluble receptor (SFlt-1) in essential hypertension [abstract]. Am J Cardiol. 2001:87: 805-7, A9)

13 Falciani M, Gori AM, Fedi S, et al. Elevated tissue factor and tissue factor pathway inhibitor circulating levels in ischaemic heart disease patients. Thromb Haemost 1998:79:495-9.

14 Battegay EJ. Angiogenesis: mechanistic insights, neovascular diseases, and therapeutic prospects. J Mol Med 1995;73:333-46.

15 Blann AD, Taberner DA. A reliable marker of endothelial cell dysfunction: does it exist? Br J Haematol 1995;90:244-8.

16 Pearson JD. Markers of endothelial perturbation and damage. $\mathrm{Br} J$ Rheumatol 1993;32:651-2.

17 Hamsten A, Walldius G, Szamosi A, et al. Relationship of angiographically defined coronary artery disease to serum lipoproteins and apolipoproteins in young survivors of myocardial infarction. Circulation 1986;73:1097-110.

18 Austen WG, Edwards JE, Frye RL, et al. A reporting system on patients evaluated for coronary artery disease. Report of the ad hoc committee for grading of coronary artery disease, Council on Cardiovascular Surgery, American Heart Association. Circulation 1975:51:5-40.

19 Machin D, Campbell M. Statistical tables for the design of clinical trials. Oxford: Blackwell Scientific, 1987.

20 Blann AD, Lip GYH. The endothelium in atherothrombotic disease: assessment of function, mechanisms and clinical implications. Blood Coagul Fibrinolysis 1998:9:297-306.

21 Giesen PL, Fyfe BS, Fallon JT, et al. Intimal tissue factor activity is released from the arterial wall after injury. Thromb Haemost 2000;83:622-8.

22 Koyama T, Ohdama S, Aoki N. Plasma tissue factor reflects endothelial cell injury rather than upregulation of tissue factor expression. Thromb Haemost 1997:78:972.

23 Misumi K, Ogawa H, Yasue H, et al. Comparison of plasma tissue factor levels in unstable and stable angina pectoris. Am J Cardiol 1998;81:22-6.

24 Fleisch M, Billinger M, Eberli FR, et al. Physiologically assessed coronary collateral flow and intracoronary growth factor concentrations in patients with 1- to 3-vessel coronary artery disease. Circulation 1999;100:1945-50.

25 Jelkmann W. Pitfalls in the measurement of circulating vascular endothelial growth factor. Clin Chem 2001;47:617-23

26 Webb NJ, Bottomley MJ, Watson CJ, et al. Vascular endothelial growth factor (VEGF) is released from platelets during blood clotting: implications for measurement of circulating VEGF levels in clinical disease. Clin Sci 1998;94:395-404

27 Yildirir A, Tokgozoglu SL, Haznedaroglu I, et al. Extent of coronary atherosclerosis and homocysteine affect endothelial markers. Angiology 2001;52:589-96.

28 Carmeliet P, Moons L, Dewerchin M, et al. Insights in vessel development and vascular disorders using targeted inactivation and transfer of vascular endothelial growth factor, the tissue factor receptor, and the plasminogen system. Ann NY Acad Sci 1997;811:191-206. 
29 Armesilla AL, Lorenzo E, Gomez A, et al. Vascular endothelial growth factor activates nuclear factor of activated T cells in human endothelial cells: a role for tissue factor gene expression. Mol Cell Biol 1999; 19:2032-43.

30 Mechtcheriakova D, Wlachos A, Holzmuller $\mathrm{H}$, et al. Vascular endothelial cell growth factor-induced tissue factor expression in endothelial cells is mediated by EGR-1. Blood 1999;93:3811-23.

31 Abe K, Shoji M, Chen J, et al. Regulation of vascular endothelial growth factor production and angiogenesis by the cytoplasmic tail of tissue factor. Proc Natl Acad Sci USA 1999:96:8663-8.

32 Chen J, Bierhaus A, Schiekofer S, et al. Tissue factor - a receptor involved in the control of cellular properties, including angiogenesis. Thromb Haemost $2001 ; 86: 334-45$.

33 Rickles FR, Shoji M, Abe K. The role of the hemostatic system in tumor growth, metastasis, and angiogenesis: tissue factor is a bifunctional molecule capable of inducing both fibrin deposition and angiogenesis in cancer. Int $\mathrm{J}$ Hematol 2001;73:145-50.

34 Hojo Y, Ikeda U, Zhu Y, et al. Expression of vascular endothelial growth factor in patients with acute myocardial infarction. J Am Coll Cardiol 2000:35:968-73.

35 Kranz A, Rau C, Kochs M, et al. Elevation of vascular endothelial growth factor-A serum levels following acute myocardial infarction. Evidence for its origin and functional significance. J Mol Cell Cardiol 2000;32:65-72.

36 Lee SH, Wolf PL, Escudero R, et al. Early expression of angiogenesis factors in acute myocardial ischemia and infarction. N Engl J Med 2000;342:626-33.

37 Soeki T, Tamura Y, Shinohara H, et al. Serial changes in serum VEGF and HGF in patients with acute myocardial infarction. Cardiology 2000;93:168-74

\section{IMAGES IN CARDIOLOGY}

\section{A case of bepridil induced interstitial pneumonitis}

W

report a case of interstitial pneumonitis during the administration of an antiarrhythmic drug, bepridil. A 65 year old man with paroxysmal atrial fibrillation and old myocardial infarction began to take $150 \mathrm{mg} /$ day of bepridil on 24 April 2002. Two weeks later, he developed cough and fever, which did not respond to oral antibiotics. He visited our clinic at one month of bepridil administration. The physical examinations revealed fine crackles in the bilateral lower lung fields. His arterial blood gas analysis showed severe hypoxia $\left(\mathrm{pO}_{2} 55 \mathrm{~mm} \mathrm{Hg}\right)$. The chest $x$ ray and the high resolution CT respectively revealed bilateral reticular shadow and micro fibrosis dominantly in the lower lung fields (upper panels). Based on the tentative diagnosis of severe interstitial pneumonitis, we started $40 \mathrm{mg} /$ day of oral prednisolone. Bepridil was stopped as a possible cause of the drug induced interstitial pneumonitis. Since his $x$ ray findings and symptoms, cough and dyspnoea, did not significantly ameliorate, high dose prednisolone, $500 \mathrm{mg}$ per day, was intravenously administered for 3 days. Then $60 \mathrm{mg}$ per day of oral prednisolone followed, which was tapered stepwise. The lymphocyte suppression test (LST) against bepridil performed on 3 June, was borderline positive $(1.6 \times)$ even under the influence of already started steroid. Peak values of KL- 6 and SP-D were $692 \mathrm{U} / \mathrm{ml}(<500)$ and $221 \mathrm{ng} / \mathrm{ml}$ $(<110)$, respectively. Numbers in the parentheses are normal ranges. His $x$ ray and CT findings (below panels) as well as symptoms responded well.

This is the first report of bepridil induced interstitial pneumonitis with the LST findings.

K Naoshi

A Teruhiko aoyagi@ve.catv.ne.jp
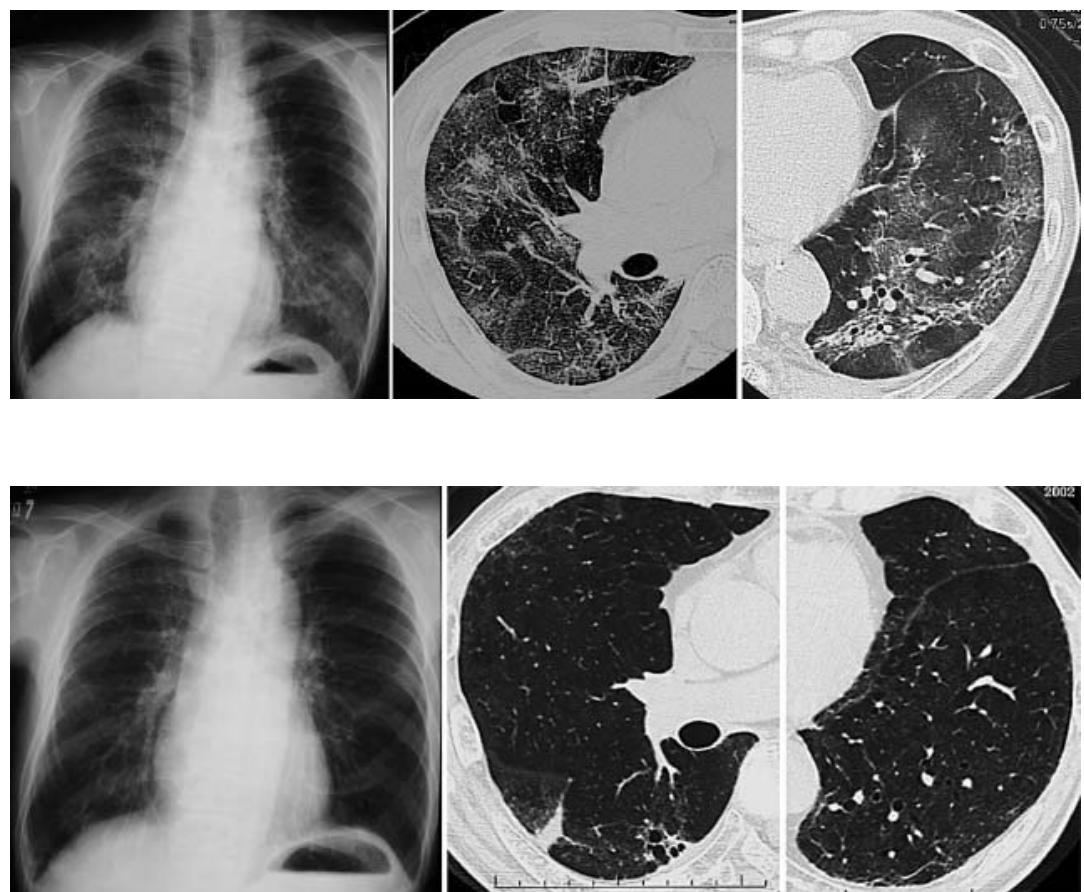